\title{
EGYPTONA: Proposal Promising Drug to Control of COVID-19 Infection
}

\author{
Tamer Ibrahim Abdel-Wahab ${ }^{1}$, Sherif Ibrahim Abdel-Wahab ${ }^{1}$, Eman Ibrahim Abdel-Wahab ${ }^{2}$ and Manal M Adel ${ }^{3 *}$ \\ ${ }^{1}$ Crop Intensification Research Department, Field Crops Research Institute, Agricultural Research Center, Giza, Egypt \\ ${ }^{2}$ Food Legumes Research Department, Field Crops Research Institute, Agricultural Research Center, Giza, Egypt \\ ${ }^{3}$ Pests and Plant Protection Department, National Research Center, Egypt
}

\begin{abstract}
EGYPTONA is a promising Egyptian treatment for COVID-19 patients. The objective of this study was to find out an active drug to control COVID-19 infection. COVID-19 virus attacks the human cell based on the levels of iron ( $\mathrm{Fe}^{2+}$ ), magnesium ( $\left.\mathrm{Mg}^{2+}\right)$ and zinc $\left(\mathrm{Zn}^{2+}\right)$ in the blood. EGYPTONA drug included four chemical compounds to biological control of COVID-19 in the human blood: hepcidin hormone regulators + inhibitors block the release of virions after budding from the host cell + mixture of magnesium chloride hexahydrate with zinc gluconate + clopidogrel bisulfate. Although the proposed antiviral drug (EGYPTONA) is a promising active drug to control of COVID-19 in the human blood, this drug needs clinical trials to determine the effective and appropriate dose of EGYPTONA drug to reduce the expected side effects.
\end{abstract}

KEYWORDS: COVID-19; Iron; Magnesium; Zinc; EGYPTONA drug

\section{INTRODUCTION}

Coronaviruses are a broad family of viruses that may cause disease in animals and humans. COVID-19 disease is an infectious disease caused by the last discovered virus of the Coronavirus strain. There was no knowledge of the existence of this new virus and its disease before the outbreak began in the Chinese city of Wuhan in December 2019. COVID -19 has now turned into a pandemic affecting many countries of the world. People can catch COVID-19 from other people who have the virus. The disease is spread mainly from person to person through small droplets that a person with COVID -19 secretes from their nose or mouth when they cough, sneeze or speak. These droplets are of relatively heavy weight, as they do not move to a faraway place, but rather fall quickly to the ground. Therefore, it is important to maintain a distance of at least 1 meter ( 3 feet) from others. These droplets may land on objects and surfaces surrounding a person, such as tables, doorknobs, and stair railings. People can then become infected when they touch these objects or surfaces and then touch their eyes, nose, or mouth. Therefore, it is important to wash hands frequently with soap and water, or to clean them with an alcohol-based hand rub disinfectant. COVID-19 infects the lungs, and the main symptoms appear fever and a persistent dry cough. The British National Health Service says a "dry cough" means a crusty cough that is not accompanied by any phlegm (thick mucus). A persistent cough means a prolonged and frequent cough for more than an hour or three or more coughing episodes within 24 hours, but if you usually suffer from a cough, the situation may be worse than usual. It also leads to shortness of breath, which is often described as severe chest tightness, difficulty breathing or a feeling of suffocation. The patient feels a fever if his temperature exceeds $37.8{ }^{\circ} \mathrm{C}$, as this leads to a feeling of warmth, cold or shivering. Sore throat, headache, and diarrhea, as well as a loss of sense of smell and taste have also been reported. It takes an average of five days for symptoms to start, but some people develop them later. The World Health Organization says that the incubation period for the virus is up to 14 days. Figure 1 shows most of the symptoms when infected with this virus are loss sense of taste and smell, loss of appetite, nausea, vomiting, blood clots, stroke, fever, respiratory mucus layer, chest pain, dry cough, sore
Quick Response Code:

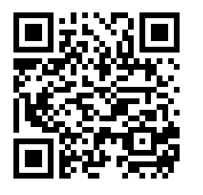

Address for correspondence: Manal M Adel, Pests and Plant Protection Department, National Research Center, Egypt

Received: September 29, $2020 \quad$ Published: October 12, 2020

How to cite this article: Abdel-Wahab TI, Abdel-Wahab Shi, Abdel-Wahab EI, Adel MM. EGYPTONA: Proposal Promising Drug to Control of COVID-19 Infection. 2020 - 2(5) OAJBS. ID.000225. DOI: $10.38125 / \mathrm{OAJBS} .000225$ 
throat, irregular heartbeats, disorders of the endocrine glands, inflammation of lymphatic system, diarrhea, malabsorption, the fatigue of the liver and heart disorders.

Figure 1 shows that infection with the virus resulted from an excess of $\mathrm{Fe}^{2+}$, and deficiency of magnesium $\left(\mathrm{Mg}^{2+}\right)$ and zinc $\left(\mathrm{Zn}^{2+}\right)$ in the blood, in addition to the new generations of the virus as a result of the virus reproduction in the blood. T.SH.E. Abdel-Wahab and M Adel (1) theory indicated that eating wild animals (bats in particular) is the main source of the flu disease's transformation into COVID-19 disease as these animals live on a lot of blood, which therefore contains high levels of $\mathrm{Fe}^{2+}$. Thus, the speed of reproduction of COVID-19 is highly dependent on the stock of $\mathrm{Fe}^{2+}$ in human blood as reported by Abdel-Wahab et al. [1]. Therefore, most countries (especially high-income countries) with a diet high in $\mathrm{Fe}^{2+}$ are the ones that have had very high injuries, as well as deaths.

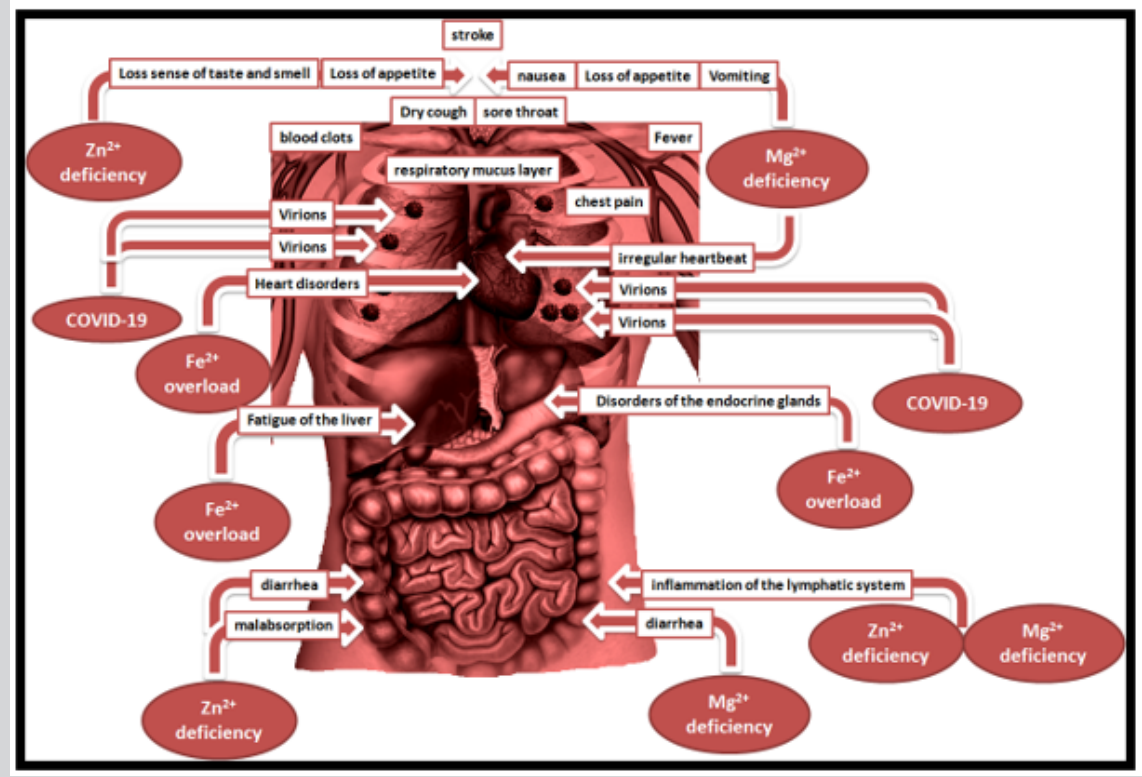

Figure 1: Schematic of COVID-19 symptoms in the human body.

$\mathrm{Mg}^{2+}$ has a strong relationship with the immune system, in both nonspecific and specific immune responses, also known as an innate and acquired immune response [2]. They added that $\mathrm{Mg}^{2+}$ is the second-most abundant cation in cellular systems. Scientific reports indicated that there is a close relationship between $\mathrm{Mg}^{2+}$ and the immune system, in that $\mathrm{Mg}^{2+}$ plays a major role in the immune response. $\mathrm{Mg}^{2+}$ is a cofactor for immunoglobulin synthesis, immune cell commitment, antibody-dependent cytolysis, ...etc. $\mathrm{Mg}^{2+}$ deficiency also seems to accelerate thymus involution. One of the most remarkable results, regarding the effects of $\mathrm{Mg}^{2+}$ deficiency on the organism, is the higher level of apoptosis shown in thymuses from Mgdeficient rats as compared with controls [3]. Understanding the normal morphological features of the thymus gland and its disorders provides a cornerstone for assessing immune system function. According to Pearse [4], the thymus, the primary lymphoid organ and the primary site for the development of the T-cell immune function, is morphologically similar across species. It is actually an epithelial organ in which epithelial cells provide a framework that contains $\mathrm{T}$ cells as well as smaller numbers of other lymphocytes. There is a symbiotic interaction between the microenvironment in the thymus and the developing T cells, and the specificity of the release of T cells into the systemic circulation is under the control of the thymus. The thymus cortex in a small animal populated by developing $\mathrm{T}$ cells with a smaller proportion of epithelial cells attached to it. Larger and more mature $\mathrm{T}$ cells are found in the medulla where the epithelial and other cell types are more abundant. Consequently, the deficiency of $\mathrm{Mg}^{2+}$ in the human body will lead directly to infection with COVID-19, even if the level of $\mathrm{Fe}^{2+}$ in the blood is normal and does not exceed the critical level. Previous studies indicated that elderly subjects are exposed to $\mathrm{Mg}^{2+}$ deficiency for insufficient intake or changes in $\mathrm{Mg} 2+$ metabolism as a result of $\mathrm{Mg}^{2+}$ deficiency contributing to the aging process and exposure to age-related diseases.

These symptoms are stress, defective membrane function, inflammation, cardiovascular disease, diabetes, and immune deficiency [5], which explains the COVID-19 attacking the elderly. It was also found that the blood of elderly people is often characterized by the deficiency of $\mathrm{Mg}^{2+}$ and vitamin $\mathrm{D}$, as strong interactions between the two nutrients have been shown in addition to their influence on the mechanisms of the immune system [6].

$\mathrm{Zn}^{2+}$ has a close relationship with the immune system of the human body. Some scientific reports showed that $\mathrm{Zn}^{2+}$ deficiency increased duration-dependently the number of total white blood cells, granulocytes (neutrophil, eosinophil and basophil) and monocytes in $2-4 \mathrm{wk}$ without changing the number of lymphocytes, T lymphocytes, B lymphocytes or NK cells. Zinc is an antioxidant and anti-inflammatory. $\mathrm{Zn}^{2+}$ also plays a very important role in the treatment of acute diarrhea in children, and vascular dermatitis, as well as the common cold. It appears that $\mathrm{Zn}^{2+}$ deficiency is prevalent in the developing world and as many as two billion subjects may be growth retarded due to zinc deficiency [7]. Trying to find out a medicinal drug is the goal for any country to treat patients and stop deaths. 
Figure 2 illustrates T.SH.E. Abdel-Wahab [1] theory which deals with a human that infected with COVID-19. This theory assumes $\mathrm{Fe}^{2+}$ overload and deficiency of $\mathrm{Mg}^{2+}$ than the normal levels of those elements in the blood are the main factors that led to the high infection of COVID-19, particularly in elderly people. Also, it is likely that this biological situation will be enhanced by a deficiency of $\mathrm{Zn}^{2+}$ than the normal level of this element in the blood. Thus, we believe that $\mathrm{Fe}^{2+}$ overload and $\mathrm{Mg}^{2+}$ deficiency in the patient's blood are the basis for increased mortality in most elderly people in developed countries. Meanwhile, the increased confirmed cases with low deaths probably were due to the normal Fe2+ level in the blood and deficiency of each of $\mathrm{Mg}^{2+}$ and $\mathrm{Zn}^{2+}$ than the normal level in the blood of most elderly people, which explains why those patients do not feel severely symptoms which reflected on shifting their clinical results from positive to negative during the fourteen days. Here, we confirm that no clinical trials have been conducted for the Egyptian drug 'EGYPTONA' and this promising drug need determine the appropriate dose to reduce the expected side effects. Therefore, the objective of this study was to find out an active drug to control COVID-19 infection.

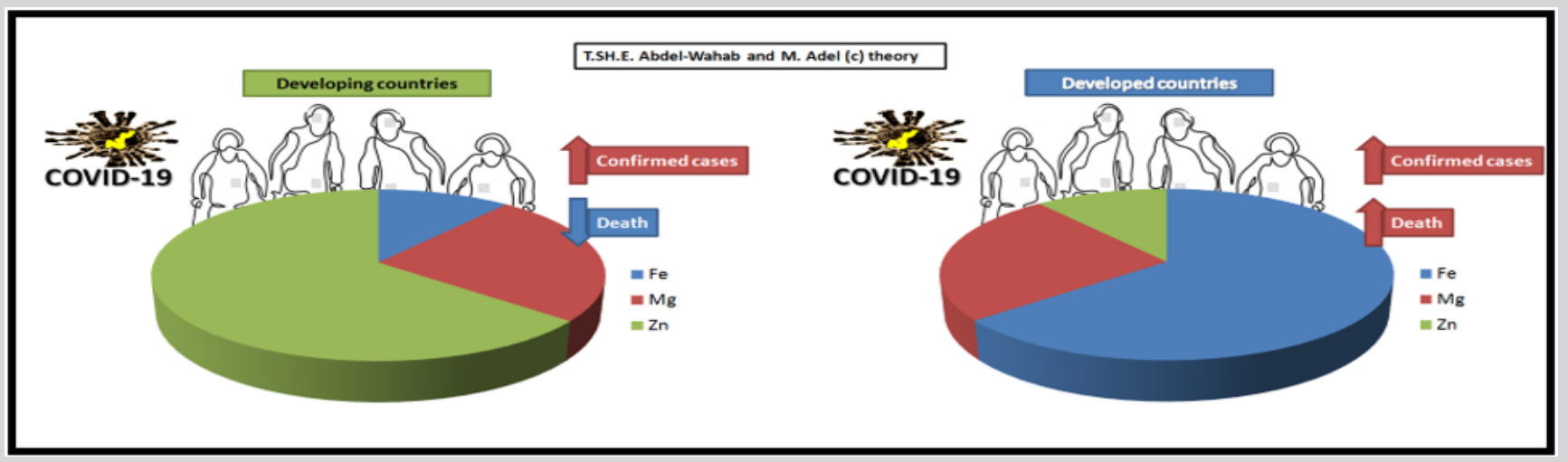

Figure 2: T.SH.E. Abdel-Wahab and M. Adel-c theory.

The Chemical Composition of The Proposed EGYPTONA Drug to Biological Control of COVID-19 In the Human Blood

Little is understood about how COVID-19 reproduces inside human host cells, spreading infection. Figure 3 shows schematic of the viral replication by blood cells to form a launching pad. The steps include attachment and entry of the virion, translation of mRNA into protein, genome replication, assembly of new particles, and release of particles from the cell. The release of new generations of the virus in the patient's blood is the main factor in harnessing the human cell to produce many generations of the virus in a very fast period of time to increase the patient's suffering and the feeling of inability to breathe. We will consider each of these steps, and then move on to a discussion of how the COVID-19 virus infects us and causes disease. Hepcidin is a peptide hormone produced by specific cells in the liver, as it is the basis for $\mathrm{Fe}^{2+}$ balance in the human body. Sometimes there is an imbalance in the liver's secretion of hepcidin, which leads to $\mathrm{Fe}^{2+}$ disturbances in the blood. In a healthy adult human, plasma $\mathrm{Fe}^{2+}$ is strictly maintained in the range of $10-30 \mu \mathrm{M}$. Longterm deviations from this range cause clinically important $\mathrm{Fe}^{2+}$ related disorders [8]. Low $\mathrm{Fe}^{2+}$ in the plasma negatively affects the amount allocated to red blood cells and thus the emergence of anemia. Here, the risk of $\mathrm{Fe}^{2+}$-protein deficiency appears, which leads to some changes in the tongue and esophagus, as well as muscle failure. While what we are interested in this context is an increase in blood $\mathrm{Fe}^{2+}$, when iron increases in the plasma, the $\mathrm{Fe}^{2+}$ will bind with organic materials such as citrate or albumin, and $\mathrm{Fe}^{2+}$ accumulates in parenchymal cells in the form of $\mathrm{Fe}^{2+}$ transferrin. $\mathrm{Fe}^{2+}$ overload, which has been stored inside the cells, generates oxygen, and consequently the failure of cells and tissues of the liver will definitely occur, and the damage may extend to the cells and tissues of the heart as well as the endocrine glands.
Magnesium $\left(\mathrm{Mg}^{2+}\right)$ is the most essential element in the human body as it is stored in the cells in the bones. Little of this element diffuses into the bloodstream. $\mathrm{Mg}^{2+}$ plays an important role in many metabolic reactions in the human body, as it has an important role in the production and storage of cellular energy, cell stability, DNA synthesis, transmission of nerve signals, bone and heart metabolism and blood pressure, the symptoms of $\mathrm{Mg}^{2+}$ deficiency are nausea and general weakness with loss of appetite, as well as numbness and muscle spasms seizures and an irregular heartbeat. Vomiting, diarrhea, or high fever resulting from a viral infection leads to the patient losing a large amount of body fluids and the occurrence of dehydration in the late stages.

Zinc $\left(\mathrm{Zn}^{2+}\right)$ is an important element for reducing inflammation, such as inflammation of the lymphatic system, and it is very important for immune function, as its deficiency leads to hair loss, lack of alertness, loss sense of taste and smell, weight loss, diarrhea, as well as loss of appetite.

Continued efforts are necessary to improve vaccines and antiviral drugs as countermeasures. $\mathrm{Fe}^{2+}$ is required for most forms of organisms, and it is the most essential element for the functions of many $\mathrm{Fe}^{2+}$ - containing proteins involved in 02 transport, cellular respiration, DNA replication, and so on. EGYPTONA (Figure 4 \& 5) included four chemical compounds to biological control of COVID-19 in the human blood: hepcidin hormone regulators + inhibitors block the release of virions after budding from the host cell + mixture of magnesium chloride hexahydrate with zinc gluconate + clopidogrel bisulfate.

With respect to hepcidin hormone regulators, $\mathrm{Fe}^{2+}$ is an essential nutrient, is required for many diverse biological processes. The role of gender in the regulation of human hepcidin gene expression in the liver is unknown. Hepcidin may play a role in gender-based differences in iron metabolism and liver diseases [9]. The absence of a defined pathway to excrete excess $\mathrm{Fe}^{2+}$ makes it essential for 
the body to regulate the amount of $\mathrm{Fe}^{2+}$ absorbed; a deficiency could lead to $\mathrm{Fe}^{2+}$ deficiency and an excess to $\mathrm{Fe}^{2+}$ overload and associated disorders such as anemia and haemochromatosis respectively. This regulation is mediated by $\mathrm{Fe}^{2+}$-regulatory hormone hepcidin [10]. Hepcidin (encoded by Hamp gene) is a 25 -amino acid peptide hormone and synthesized in hepatocytes. They added that hepcidin binds to the only known $\mathrm{Fe}^{2+}$ export protein, ferroprotein (FPN), inducing its internalization and degradation, thus limiting the amount of $\mathrm{Fe}^{2+}$ released into the blood. They added that the major factors that are implicated in hepcidin regulation include $\mathrm{Fe}^{2+}$ stores, hypoxia, inflammation, and erythropoiesis.

The required $\mathrm{Fe}^{2+}$ is guaranteed by transferrin (Tf)-bound $\mathrm{Fe}^{2+}$ in human cells, which is imported into cells through receptormediated endocytosis [11]. In the endosome, Tf-bound $\mathrm{Fe}^{2+}$ is released as ferrous ion, which is translocated via divalent metal transporter 1 (DMT1) into cytoplasm where it is sequestered by ferritin (Ftn). Ftn, the major $\mathrm{Fe}^{2+}$ storage protein, composed by 24 subunits, possesses ferroxidase activity and a large cavity where up to 4500 ferric ions, as oxy-hydroxide micelles, are sequestered. The release of $\mathrm{Fe}^{2+}$ from this protein to cytoplasm occurs after reduction of ferric to ferrous ions. Then, ferrous ions are exported into plasma by ferroportin (Fpn), the only known mammalian $\mathrm{Fe}^{2+}$ exporter found on the cytoplasmic membrane of enterocytes, hepatocytes, macrophages, and placental cells. According to Rosa et al. [12], human lactoferrin (hLf), an $\mathrm{Fe}^{2+}$-binding multifunctional cationic glycoprotein secreted by exocrine glands and by neutrophils, is a key element of host defenses. Bovine Lf (bLf) inhibits intracellular $\mathrm{Fe}^{2+}$ overload, an unsafe condition enhancing in vivo susceptibility to infections, as well as anemia of inflammation (AI), reestablishing inflammatory homeostasis. Liu et al. [13] reported that disorders of $\mathrm{Fe}^{2+}$ metabolism are associated with diverse diseases, including anemias (e.g., $\mathrm{Fe}^{2+}$ - deficiency anemia and anemia of chronic diseases) and $\mathrm{Fe}^{2+}$ overload diseases, such as hereditary hemochromatosis and $\beta$-thalassemia. Hepcidin (encoded by Hamp gene) is a peptide hormone synthesized by hepatocytes, and it plays an important role in regulating the systematic $\mathrm{Fe}^{2+}$ homeostasis. As the systemic $\mathrm{Fe}^{2+}$ regulator, hepcidin, not only controls dietary $\mathrm{Fe}^{2+}$ absorption and $\mathrm{Fe}^{2+}$ egress out of $\mathrm{Fe}^{2+}$ storage cells, but also induces $\mathrm{Fe}^{2+}$ redistribution in various organs.

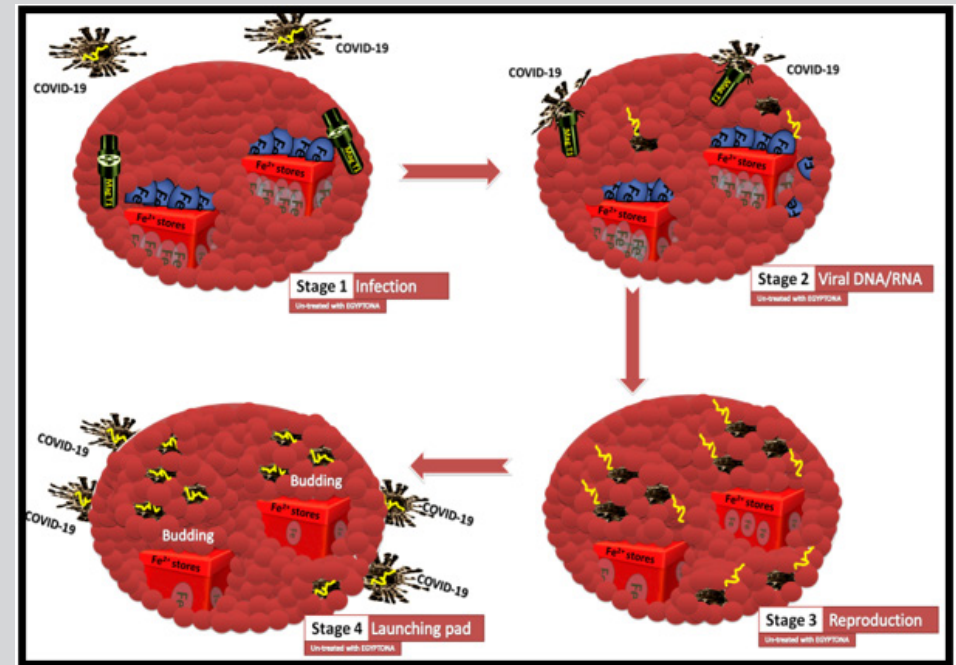

Figure 3: Schematic of the viral replication by blood cells.

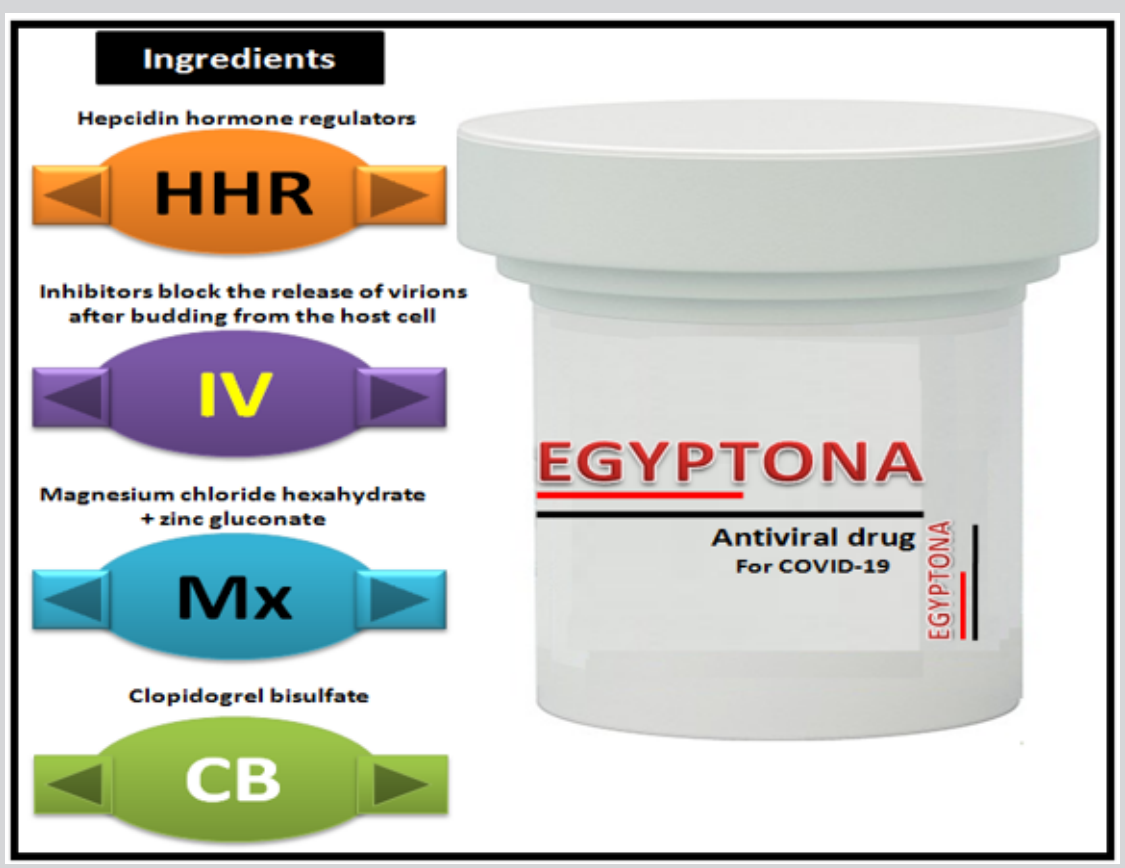

Figure 4: Schematic of the chemical components of EGYPTONA drug. 


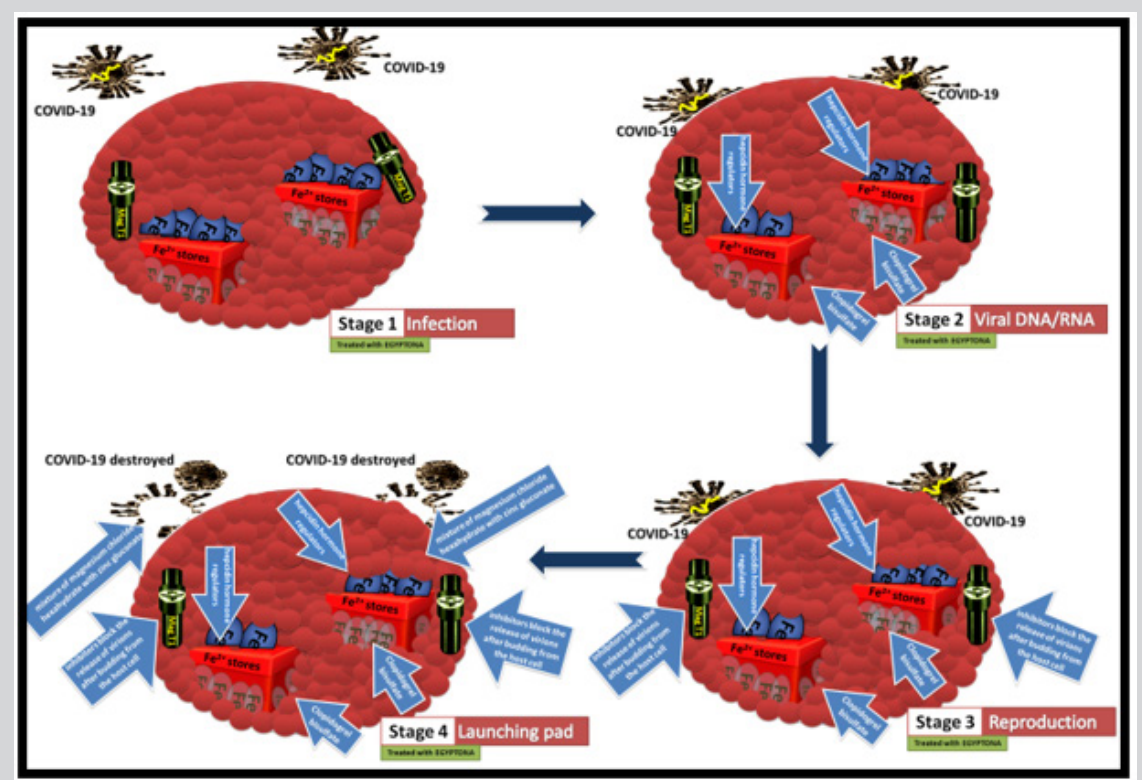

Figure 5: Schematic of the biological control of COVID-19 in the blood by EGYPTONA drug.

By directly binding to the extracellular domain of ferroportin, hepcidin induces endocytosis and degradation of the transmembrane protein, thereby preventing $\mathrm{Fe}^{2+}$ egress from the cell [14]. High levels of ferroportin are found in enterocytes in the duodenum (to transportabsorbed $\mathrm{Fe}^{2+}$ ), in hepatocytes (to transport stored $\mathrm{Fe}^{2+}$ ), and in macrophages (to transport recycled $\mathrm{Fe}^{2+}$ ), which together control systemic $\mathrm{Fe}^{2+}$ levels [11]. It is important to mention that ferroprotin is found in the lung [15] which leads to difficulty breathing. By reducing surface ferroportin, the expression of hepcidin limits the absorption, remobilization and recycling of $\mathrm{Fe}^{2+}$, thereby reducing $\mathrm{Fe}^{2+}$ plasma levels. That is why hepcidin hormone regulators are the main chemical component of the Egyptian drug (EGYPTONA).

With respect to inhibitors block the release of virions after budding from the host cell, only two classes of drugs are currently approved for the treatment of influenza: M2 ion channel blockers (adamantanes) and neuraminidase (NA) inhibitors [16]. Adamantanes inhibit FluA replication by blocking virus entry. However, they have no activity against FluB viruses, are often associated with serious side effects, and suffer from rapid emergence of drug-resistant viruses [17]. NA inhibitors block the release of virions after budding from the host cell [18]. They exhibit activity against both FluA and FluB viruses. So, we expected that these inhibitors will be relatively effective in controlling COVID-19 where COVID-19 RNA polymerase is a heterotrimeric complex of three virus-encoded proteins that essential for viral RNA synthesis and their interactions are essential for polymerase function. Thus, inhibition of these interactions represents an attractive strategy for the development of EGYPTONA drug with broad efficacy against all influenza virus strains. In other words, it is expected that EGYPTONA drug will block the release of new virions during the budding stage in the human cell.

With respect to mixture of magnesium chloride hexahydrate + zinc gluconate as we mentioned before, arms of the COVID-19 are glycoprotein. So, overexpression of P-glycoprotein in the plasma membrane causes resistance to the toxic effects of a wide variety of chemically unrelated drugs, i.e. multidrug resistance [19]. This indicates the difficulty of drug resistance to COVID-19 when it multiplies in large numbers in human blood. With respect to $\mathrm{Mg}^{2+}$ salts: two mammalian proteins, currently annotated as $\mathrm{Mg}^{2+}$ transporter 1 (MagT1; formerly IAP) and tumor suppressor candidate 3 (TUSC3; formerly N33) were initially proposed to be OST subunits based upon homology to the yeast Ost3 and Ost6 proteins [20,21]. The mechanistic role of MagT1 and TUSC3 in protein $\mathrm{N}$-glycosylation has been difficult to biochemically evaluate as these proteins dissociate from the canine OST during purification and are dispensable for glycosylation of synthetic peptide substrates [21]. MagT1 and TUSC3 are required for magnesium uptake by vertebrate cells [22]. MagT1-deficient human lymphocytes display altered kinetics of $\mathrm{Mg}^{2+}$ uptake but have normal cellular levels of $\mathrm{Mg}^{2+}$ [23]. The predominant form of MagT1 in vivo is oxidized, which is consistent with transient formation of mixed disulfides between MagT1 and a glycoprotein substrate to facilitate access of STT3B to unmodified acceptor sites [24]. They added that MagT1 depletion reduces glycosylation of STT3B-dependent substrates where HeLa cells treated with MagT1 siRNA typically showed a two- to threefold reduction in MagT1 content after 72h. Also, they found that immunofluorescence microscopy of permeabilized cells showed a reticular staining pattern for endogenous MagT1 that surrounds the nucleus and does not colocalize with the $\mathrm{Na}+$ $\mathrm{K}+$-ATPase. Thus, there is a defect in the different cellular needs. Much energy is stored in the ionic gradients across the plasma membrane, and the steep $\mathrm{Na}+$ and $\mathrm{K}+$ gradients in animal cells are used to facilitate secondary transport of molecules (sugars, neurotransmitters, amino acids, metabolites) and other ions ( $\mathrm{H}+$, $\mathrm{Ca} 2+, \mathrm{Cl}-$ ). The ion gradients are also used for rapid signaling by opening of $\mathrm{Na}+$ or $\mathrm{K}+$ selective channels in the plasma membrane in response to extracellular signals or the membrane potential [25]. Many organs use $\mathrm{Na}+$ and $\mathrm{K}+$ gradients for their specialized functions. In the kidneys, the Na, K-ATPase is highly expressed, an estimate says up to 50 million pumps per cell in the distal convoluted tubule [26], because $\mathrm{Na}+$ gradient is utilized by the main kidney functions, to filter the blood of waste products, to reabsorb glucose and amino acids, to regulate electrolytes and to maintain $\mathrm{pH}$. In humans, loss-of-function mutations in the MagT1 gene cause $\mathrm{X}$-linked magnesium deficiency with Epstein-Barr virus (EBV) infection and neoplasia (XMEN), a disease that has a broad range of clinical and immunological consequences. In other word, loss-offunction mutations in MagT1 cause an immune deficiency named 
'XMEN syndrome', characterized by CD4 lymphopenia, chronic EBV infection, and EBV-related lymphoproliferative disorders [27]. With respect to TUSC3, it is an indispensable member of the vertebrate plasma membrane $\mathrm{Mg}^{2+}$ ion transport system [22]. It is an $\mathrm{Mg}^{2+}$ transporter involved in $\mathrm{Mg}^{2+}$ transport and homeostasis, which is important for learning and memory, embryonic development and testis maturation [28]. They added that dysfunction or deletion of TUSC3 exerts its oncological effects as a modulator by inhibiting glycosylation efficiency and consequently inducing endoplasmic reticulum stress and malignant cell transformation.

$\mathrm{Mg}^{2+}$ is very important for the normal functioning of cells, nerves, muscles, bones, and the heart. Usually, a well-balanced diet provides normal blood levels of $\mathrm{Mg}^{2+}$. Low $\mathrm{Mg}^{2+}$ levels in the body have been linked to diseases such as osteoporosis, high blood pressure, clogged arteries, hereditary heart disease, diabetes, and stroke. This medication is a mineral supplement used when certain situations cause your body to lose $\mathrm{Mg}^{2+}$ faster than you can replace it from your diet. These situations include a poor diet, alcoholism, or other medical conditions (e.g., severe diarrhea/vomiting, stomach/ intestinal absorption problems, poorly controlled diabetes). As a result of contact of the virus arms with the blood cell, an imbalance of the proteins transporting $\mathrm{Mg}^{2+}$ in the patient's blood leads to tumors of the lymphatic system in the patient. Hence, it is likely that the use of $\mathrm{Mg}^{2+}$ salts is one of the beneficial treatments for the virus patients, as it works to disperse COVID-19 by its connection with the $\mathrm{Mg}^{2+}$ salts that lead to the loss of the virus arms and thereby not focus on red blood cells, especially Sugimoto [29] suggested that excessive oral intake of $\mathrm{MgO}$ can also induce $\mathrm{Fe}^{2+}$ deficiency anemia.

It was shown that the decrease of extracellular space volume evoked by electrical stimulation was indeed accompanied by an increase in $\mathrm{Cl}$ - that started to change only after the stimulation [30]. The $\mathrm{Cl}$ - conductance determines the extent of volume changes because $\mathrm{Cl}$ - must follow the changes of membrane potential, which inevitably leads to changes in cell volume [31]. He added that this voltage-evoked Cl--dependent volume change does not affect intracellular cation concentrations or the amount of energy that is necessary to support the system.

Glycans are usually found on the cells in the form of glycoproteins or glycolipids, where they are covalently attached to either proteins or lipids, respectively. Since glycans play a part in almost all biological processes such as intra- and intercellular signaling, organ development, immunological responses, tumor growth, and even stability of bioconjugates, a comprehensive analysis of cellular glycan repertoire is essential for the study of underlying mechanisms in these complex biological processes [32]. They added that both glycomics and glycoproteomics analysis involves cleavage of the glycoprotein into smaller peptides by protease(s).

In order to favor complete protease digestion, disulfide bridges in the protein are broken by reduction with tris (2-carboxyethyl) phosphine (TCEP), or 2-mercaptoethanol The reduction is usually followed by alkylation with iodoacetamide ("carbamidomethylation") or iodoacetic acid ("carboxymethylation") to prevent reformation of disulfide bond.

The protease digestion is usually done by a protease enzyme or combination of enzymes, such as trypsin, Glu-C, chymotrypsin, etc., which should be selected based on the protein sequence of the target protein if the sequence is available [33]. High amount of sodium hydroxide $(\mathrm{NaOH})$ and unreacted methyl iodide (CH3I) are present in the final reaction mixture [34]. These adversely influence the MS ionization efficiency of the permthylated glycans, thus rendering their analysis quite impossible. The use of hydroxide ( $\mathrm{OH}-)$ played an important role in separating the virus arms from the blood cells and thus the failure of the docking process and by using $\mathrm{OH}$ -

Subclinical vitamin A deficiency renders 250,000-500,000 children blind annually, while $\mathrm{Zn}^{2+}$ deficiency increases the risk of death from diarrhea, malaria, and respiratory disease [35]. $\mathrm{Zn}^{2+}$ deficiency has been suggested as a risk factor with adverse longterm effects on growth, immunity, and metabolic status of surviving offspring [36]. It is important to mention that antiviral activity of $\mathrm{Zn}^{2+}$ oxide nanoparticles (ZnO-NPs) and PEGylated $\mathrm{Zn}^{2+}$ oxide nanoparticles could be a novel, effective, and promising antiviral agent against H1N1 influenza virus infection [37].

National Center for Complementary and Integrative Health showed that $\mathrm{Zn}^{2+}$ is available in two forms oral $\mathrm{Zn}^{2+}$ (e.g., lozenges, tablets, syrup) and intranasal $\mathrm{Zn}^{2+}$ (e.g., swabs and gels). A 2015 analysis of clinical trials found that oral $\mathrm{Zn}^{2+}$ helps to reduce the length of colds when taken within 24 hours after symptoms start. Intranasal $\mathrm{Zn}^{2+}$ has been linked to a severe side effect (irreversible loss of the sense of smell) and should not be used. Side effects of oral $\mathrm{Zn}^{2+}$ are nausea and other gastrointestinal symptoms.

Long-term use of $\mathrm{Zn}^{2+}$, especially in high doses, can cause problems such as $\mathrm{Cu}^{2+}$ deficiency. $\mathrm{Zn}^{2+}$ may interact with drugs, including antibiotics and penicillamine (a drug used to treat rheumatoid arthritis). $\mathrm{Cu}^{2+}$ deficiency has been reported as a consequence of long-term $\mathrm{Zn}^{2+}$ supplementation [38], although a six-week experiment in which $150 \mathrm{mg} /$ day of $\mathrm{Zn}^{2+}$ was administered found no effect on plasma $\mathrm{Cu}^{2+}$ levels [39] due to the $\mathrm{Zn}^{2+}$ and $\mathrm{Cu}^{2+}$ interaction. $\mathrm{Zn}^{2+}$ is an important source for growth and for the development and health of body tissues. Studies show that $\mathrm{Zn}^{2+}$ may be better absorbed in humans in the gluconate form [40].

Zinc gluconate is used to treat and to prevent zinc deficiency. Zinc gluconate is a zinc salt of gluconic acid comprised of two gluconic acid molecules for each zinc cation $(2+)$. It is available as a trace mineral supplement and over the counter as a lozenge form for a reduced duration of common colds and with decreased symptom severity. Interestingly, zinc supplementation has become a critical intervention for treating diarrheal episodes in children. So, we suggest that magnesium chloride hexahydrate mixing with zinc gluconate is one of the essential chemical components for EGYPTONA drug to control COVID-19.

With respect to clopidogrel bisulfate, clopidogrel prevents blood clots by irreversibly binding to the P2Y12 receptor on platelets, preventing adenosine diphosphate from activating platelets. It belongs to a class of drugs called P2Y12 inhibitors. Clopidogrel bisulfate does not cause serious reductions of white cells in the blood. Clopidogrel is a prodrug of a platelet inhibitor used to reduce the risk of myocardial infarction and stroke [41]

Clopidogrel is indicated to reduce the risk of myocardialinfarction for patients with non-ST elevated acute coronary syndrome, patients with ST-elevated myocardial infarction, and in recent MI, stroke, or established peripheral arterial disease. So, clopidogrel bisulfate is an essential chemical component for EGYPTONA drug. Continued efforts are necessary to improve vaccines and anti-viral drugs as countermeasures [42]. $\mathrm{Fe}^{2+}$ is required for most forms of organisms, and it is the most essential element for the functions of many $\mathrm{Fe}^{2+}$ - containing proteins involved in $\mathrm{O}_{2}$ transport, cellular respiration, DNA replication, and so on. 
The Expected Results by Control of Fe2+ Overload in The Blood by EGYPTONA Drug

Firstly, the patient undergoes blood tests to know the level of $\mathrm{Fe}^{2+}$ in the blood, such as "serum ferritin" and "serum transferrin saturation" to check the degree of $\mathrm{Fe}^{2+}$ concentration. Secondary, it is expected that patient with $\mathrm{Fe}^{2+}$ overload will be reduced by EGYPTONA drug via the following steps as shown in Figure $5 \& 6$ :

a. Hepcidin hormone regulator bind with ferroprotein (FPN) to induce its internalization and degradation, thus limiting the amount of $\mathrm{Fe}^{2+}$ released into the blood.

b. Hepcidin hormone regulator reacts with $\mathrm{Fe}^{2+}$ to treat fatigue of the liver and disorders of the endocrine glands.

c. Inhibitors that block the release of virions after budding from the host cell to decrease virions.

d. Zinc gluconate and magnesium chloride hexahydrate react with $\mathrm{Mg}^{2+}$ deficiency to treat diarrhea.

e. Hepcidin hormone regulator + zinc gluconate and magnesium chloride hexahydrate + inhibitors that block the release of virions after budding from the host cell + clopidogrel bisulfate react with $\mathrm{Zn}^{2+}$ and $\mathrm{Mg}^{2+}$ deficiencies to treat inflammation of the lymphatic system.

f. Zinc gluconate and magnesium chloride hexahydrate react with $\mathrm{Zn}^{2+}$ deficiency to treat diarrhea, malabsorption, less sense of taste and smell and loss of appetite.

g. Hepcidin hormone regulator + clopidogrel bisulfate react with $\mathrm{Fe}^{2+}$ to treat heart disorders.

h. Clopidogrel bisulfate to treat chest pain and stroke.

i. Zinc gluconate and magnesium chloride hexahydrate + clopidogrel bisulfate react with $\mathrm{Mg}^{2+}$ deficiency to treat irregular heartbeats, vomiting and blood clots.

j. $\quad$ Zinc gluconate and magnesium chloride hexahydrate + clopidogrel bisulfate to treat fever.

k. Hepcidin hormone regulator + zinc gluconate and magnesium chloride hexahydrate + inhibitors that block the release of virions after budding from the host cell + clopidogrel bisulfate treat nausea, respiratory mucus layer, sore throat and dry cough.

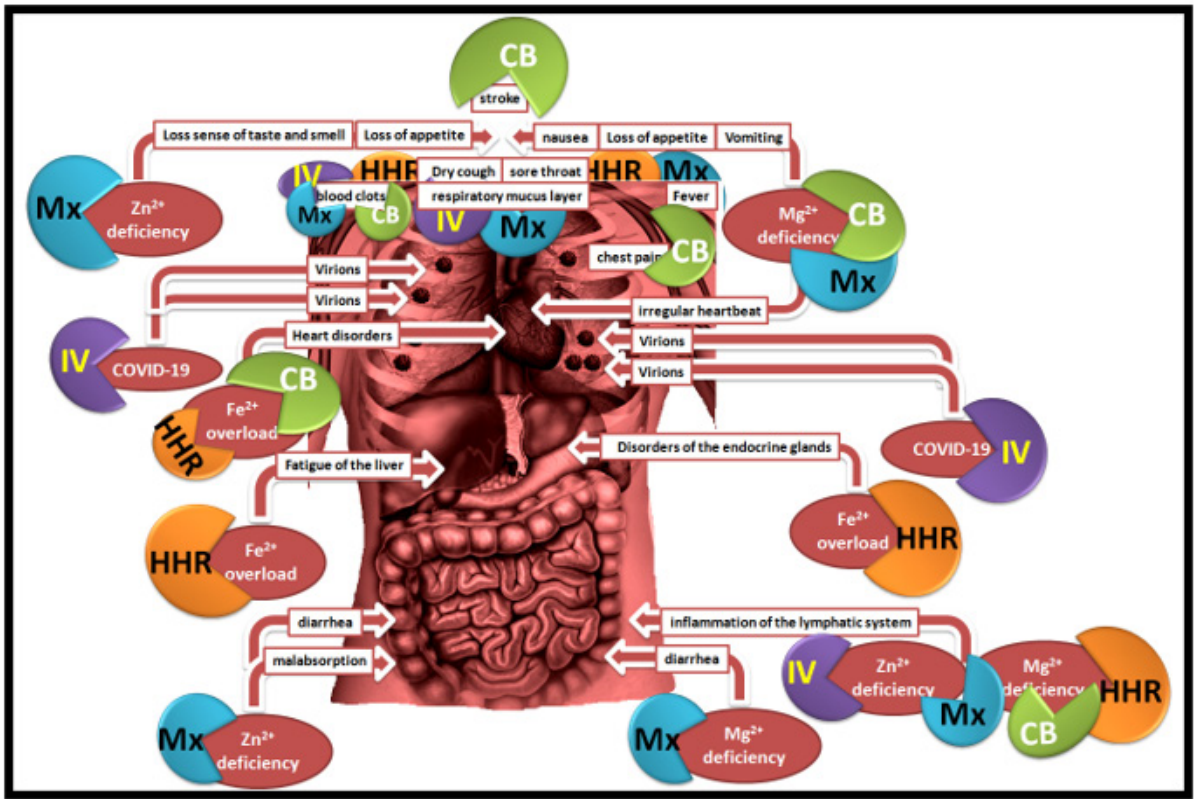

Figure 6: Schematic of role of the chemical constitutes of EGYPTONA drug.

\section{CONCLUSION}

It can be concluded that the proposed antiviral drug (EGYPTONA) that included hepcidin hormone regulators + inhibitors block the release of virions after budding from the host cell + mixture of magnesium chloride hexahydrate with zinc gluconate + clopidogrel bisulfate should be treated medically with the patient. However, analyzes and clinical trials are necessary to determine the effective and appropriate dose of EGYPTONA drug to reduce the expected side effects.

\section{REFERENCES}

1. Abdel Wahab TI, Abdel Wahab ShI, Abdel Wahab Eman I, Adel Manal M (2020) A step forward to control of COVID-19. International Journal of Pharmaceutical Research 12 (Suppl Issue 1): 1167-1190.

2. Tam M, Gómez S, González Gross M (2003) Possible roles of magnesium on the immune system. Eur J Clin Nutr 57: 1193-1197.

3. Malpuech Brugère C, Nowacki W, Gueux E, Kuryszko J, Rock E (1999) Accelerated thymus involution in magnesium deficient rats is related to enhanced apoptosis and sensitivity to oxidative stress. Br J Nutr 81: 405-411.

4. Pearse G (2006) Normal structure, function and histology of the thymus. Toxicologic Pathology 34: 504-514.

5. Rayssiguier Y, Durlach J, Guex E, Rock E, Mazur A (1993) Magnesium and aging I. Experimental data: importance of oxidative damage. Magnesium Res 6: 369-378.

6. Mc Coy H, Kenney MA (1996) Interactions between magnesium and vitamin D: possible implications in the immune system. Magnesium Res 9: 185-203.

7. Prasad AS (2008) Zinc in human health: Effect of zinc on immune cells. Mol Med, 14(5-6): 353-357. 
8. Ganz T, Nemeth E (2012) Iron metabolism: interactions with normal and disordered erythropoiesis. Cold Spring Harb Prospect Med 2(5): a011668.

9. Harrison Findik DD (2010) Gender related variations in iron metabolism and liver diseases. World J Hepatol 2 (8): 302-310.

10. Rishi G, Wallace DF, Subramaniam VN (2015) Hepcidin: Regulation of the master iron regulator. Biosci Rep 35 (3): e00192.

11. Donovan A, Lima CA, Pinkus JL, Pinkus GS, Zon LI, Robine S, Andrews NC (2005) The iron exporter ferroportin/Slc40a1 is essential for iron homeostasis. Cell Metab 1:191-200.

12. Rosa L, Cutone A, Lepanto MS, Paesano R, Valenti P (2017) Lactoferrin: A natural glycoprotein involved in iron and inflammatory homeostasis. Int J Mol Sci 18(9): 1985.

13. Liu J, Sun B, Yin H, Liu S (2016) Hepcidin: A promising therapeutic target for iron disorders. Medicine (Baltimore) 95(14): e3150.

14. Nemeth E, Tuttle MS, Powelson J, Vaughn MB, Donovan A (2004) Hepcidin regulates cellular iron efflux by binding to ferroprotein and inducing its internalization. Science 306(5704): 2090-2093.

15. Yang F, Haile DJ, Wang X, Dailey LA, Stonehuerner JG, et al. (2005) Apical location of ferroportin 1 in airway epithelia and its role in iron detoxification in the lung. Am. J Physiol Lung Cell Mol Physiol 289: L14L23.

16. De Clercq E (2006) Antiviral agents active against influenza A viruses. Nat Rev Drug Discov 5: 1015- 1025.

17. Hayden FG, Hay AJ (1992) Emergence and transmission of influenza A viruses resistant to amantadine and rimantadine. Curr Top Microbiol Immunol 176: 119-130.

18. Colman PM, Varghese JN, Laver WG (1983) Structure of the catalytic and antigenic sites in influenza virus neuraminidase. Nature 303: 41-44

19. Gottesman MM, Pastan I (1993) Biochemistry of multidrug resistance mediated by the multidrug transporter. Annu Rev Biochem 62: 385-427.

20. Mac Grogan D, Levy A, Bova GS, Isaacs WB, Bookstein R (1996) Structure and methylation-associated silencing of a gene within a homozygously deleted region of human chromosome band 8p22. Genomics 35: 55-65.

21. Kelleher DJ, Karaoglu D, Mandon EC, Gilmore R (2003) Oligosaccharyl transferase isoforms that contain different catalytic STT3 subunits have distinct enzymatic properties. Mol Cell 12: 101-111.

22. Zhou H, Clapham DE (2009) Mammalian MagT1 and TUSC3 are required for cellular magnesium uptake and vertebrate embryonic development. Proc. Natl Acad Sci USA, 106:15750 - 15755.

23. Li FY, Chaigne Delalande B, Kanellopoulou C, Davis JC, Matthews HF et al. (2011) Second messenger role for $\mathrm{Mg}^{2+}$ revealed by human T-cell immunodeficiency. Nature 475: 471-476.

24. Cherepanova NA, Shrimal S Gilmore R (2014) Oxidoreductase activity is necessary for $\mathrm{N}$-glycosylation of cysteine-proximal acceptor sites in glycoproteins. J Cell Biol Vol 206(4): 525-539.

25. Clausen MV, Hilbers F, Poulsen H (2017) The structure and function of the Na, K-ATPase isoforms in health and disease. Front Physiol 06.

26. El Mernissi G, Doucet A (1984) Quantitation of [3H] ouabain binding and turnover of Na-K-ATPase along the rabbit nephron. Am J Physiol 247 F158-F167.
27. Ravell J, Chaigne Delalande B, Lenardo M (2014) X-linked immunodeficiency with magnesium defect, Epstein-Barr virus infection, and neoplasia disease: A combined immune deficiency with magnesium defect. Curr Opin Pediatr 26 (6): 713-719.

28. Yu X, Zhai C, Fan Y, Zhang J, Liang N, et al. (2017) TUSC3: a novel tumour suppressor gene and its functional implications. J Cell Mol Med 21(9): 1711-1718.

29. Sugimoto H, Yamada U (2019) Iron deficiency anemia induced by magnesium overuse: a case report. Biopsychosoc Med 13: 18.

30. Dietzel I, Heinemann U, Hofmeier G, Lux H (1982) Stimulus-induced changes in extracellular $\mathrm{Na}+$ and $\mathrm{Cl}^{-}$concentratio in relation to changes in the size of the extracellular space. Experimental Brain Research 46 (1): $73-84$.

31. Dmitriev AV (2019) The logic of ionic homeostasis: Cations are for voltage, but not for volume. PLoS Comput. Biol 15(3): e1006894.

32. Shajahan A, Heiss C, Ishihara M, Azadi P (2017) Glycomic and glycoproteomic analysis of glycoproteins a tutorial. Anal Bioanal Chem, 409(19): 4483-4505.

33. Chen R, Jiang X, Sun D, Han G, Wang F, et al. (2009) Glycoproteomics analysis of human liver tissue by combination of multiple enzyme digestion and hydrazide chemistry. J Proteome Res 8: 651-661.

34. Desantos Garcia JL, Khalil SI, Hussein A, Hu Y, Mechref Y (2011) Enhanced sensitivity of LC-MS analysis of permethylated N-glycans through online purification. Electrophoresis 32(24): 3516-3525.

35. Tan Z, Ma G, Lin L, Liu C, Liu Y, et al. (2002) Prevalence of subclinical vitamin A deficiency and its affecting factors in 8669 children of China. Zhonghua Yu Fang Yi Xue Za Zhi 36(3): 161-163.

36. Brown KH, Rivera JA, Bhutta Z, Gibson RS, King JC, et al. (2004) International Zinc Nutrition Consultative Group (IZiNCG) technical document \#1. Assessment of the risk of zinc deficiency in populations and options for its control. Food Nutr Bull 25 (Suppl. 2): S99-S203.

37. Ghaffari H, Tavakoli A, Moradi A, Tabarraei A, Bokharaei Salim F, et al. (2019) Inhibition of H1N1 influenza virus infection by zinc oxide nanoparticles: another emerging application of nanomedicine. J Biomed Sci 26: 70

38. Prasad AS, Brewer GJ, Schoomaker EB, Rabbani P (1978) Hypocupremia induced by zinc therapy in adults. JAMA 240(20): 2166-2168.

39. Samman S, Roberts DC (1987) The effect of zinc supplements on plasma zinc and copper levels and the reported symptoms in healthy volunteers. Medical Journal of Australia. 146(5): 246-249.

40. Siepmann M, Spank S, Kluge A, Schappach A, Kirch W (2005) The pharmacokinetics of zinc from zinc gluconate: A comparison with zinc oxide in healthy men. Int. J Clin Pharmacol Ther 43(12): 562-565.

41. Zhang YJ, Li MP, Tang J, Chen XP (2017) Pharmacokinetic and pharmacodynamic responses to clopidogrel: Evidences and perspectives. Int J Environ Res Public Health 14(3): 301.

42. Lambert LC, Fauci AS (2010) Influenza vaccines for the future. The New England journal of medicine 363: 2036-2044. 